\title{
OPEN Association of gamma-glutamyl transferase with subclinical coronary atherosclerosis and cardiac outcomes in non-alcoholics
}

\begin{abstract}
Yong-Giun Kim ${ }^{1}$, Gyung-Min Park ${ }^{1 凶}$, Seung Bum Lee ${ }^{2 \bowtie}$, Dong Hyun Yang ${ }^{3}$, Joon-Won Kang ${ }^{3}$, Tae-Hwan Lim ${ }^{3}$, Hong-Kyu Kim ${ }^{4}$, Jaewon Choe ${ }^{4}$, Seung-Whan Lee ${ }^{5}$ \&oung-Hak Kim ${ }^{5}$

In an asymptomatic population, we determined the relationship between serum gamma-glutamyl transferase (GGT) and subclinical atherosclerosis, using coronary computed tomography angiography (CCTA). This was a retrospective observational cohort study which analyzed 5120 consecutive asymptomatic individuals with no prior history of coronary artery disease or significant alcohol intake who voluntarily underwent CCTA as part of a general health examination. All subjects were stratified into tertiles based on GGT levels. Degree and extent of subclinical coronary atherosclerosis were evaluated using CCTA. Cardiac events were a composite of all-cause death, myocardial infarction, unstable angina, and coronary revascularization. After adjustment for cardiovascular risk factors, there were no significant differences among GGT tertiles in terms of adjusted odds ratios for noncalcified and mixed plaques. The risk of any atherosclerotic and calcified plaques, significant stenosis, multi-vessel disease, and significant stenosis in the left main or proximal left anterior descending artery was higher in the third GGT tertile than in the first tertile (all $p<0.05$ ). Over a median 5.4year follow-up, the third GGT tertile had significant adjusted hazards ratios for cardiac events than did the first GGT tertile, even after stepwise adjustment for cardiovascular risk factors (all $p<0.01$ ). In asymptomatic individuals, elevated GGT was independently associated with high-risk feature atherosclerosis and poorer cardiac outcomes.
\end{abstract}

Gamma-glutamyl transferase (GGT) is a hepatobiliary enzyme synthesized in epithelial cells of the intrahepatic duct. Elevated GGT is a specific indicator of obstructive biliary diseases and excessive alcohol intake ${ }^{1}$. An association between serum GGT and nonalcoholic fatty liver disease (NAFLD) has been established, and some authors consider serum GGT as a surrogate marker of NAFLD ${ }^{2,3}$. Several studies have reported that serum GGT levels are related to cardiovascular diseases $(\mathrm{CVD})^{4-6}$. Based on studies to date, it may be assumed that GGT represents a link between NAFLD and atherosclerosis, with insulin resistance as a common pathophysiology ${ }^{7-9}$. Recently, coronary computed tomography angiography (CCTA) has proven to be effective in providing a comprehensive evaluation of coronary atherosclerosis ${ }^{10}$. An association of elevated serum GGT and subclinical coronary atherosclerosis detected by CCTA has also been reported ${ }^{11,12}$. However, there are limited data on clinical outcomes including future cardiac events. Therefore, the present study sought to evaluate the relationship between serum GGT and subclinical coronary atherosclerosis as well as cardiac events in a large cohort of asymptomatic Korean individuals who voluntarily underwent CCTA for early detection of coronary artery disease (CAD).

\footnotetext{
${ }^{1}$ Department of Cardiology, Ulsan University Hospital, University of Ulsan College of Medicine, 877, Bangeojinsunhwan-doro, Dong-gu, Ulsan 44033, Republic of Korea. ${ }^{2}$ Department of Gastroenterology and Hepatology, Ulsan University Hospital, University of Ulsan College of Medicine, 877 Bangeojinsunhwando-ro, Dong-gu, Ulsan 44033, Republic of Korea. ${ }^{3}$ Department of Radiology, Asan Medical Center, University of Ulsan College of Medicine, Seoul, Republic of Korea. ${ }^{4}$ Department of Health Screening and Promotion Center, Asan Medical Center, University of Ulsan College of Medicine, Seoul, Republic of Korea. ${ }^{5}$ Department of Cardiology, Asan Medical Center, University of Ulsan College of Medicine, Seoul, Republic of Korea. ${ }^{\square}$ email: min8684@ hanmail.net; southbay148@naver.com
} 


\begin{tabular}{|c|c|c|c|c|c|}
\hline \multirow[b]{2}{*}{ Characteristics } & \multirow[b]{2}{*}{$\begin{array}{l}\text { Overall } \\
(n=5,120)\end{array}$} & \multicolumn{3}{|c|}{ Gamma-glutamyl transferase } & \multirow[b]{2}{*}{$p$ value } \\
\hline & & $\begin{array}{l}\text { Tertile } 1 \\
\leq 15 \text { IU/L }(n=1618)\end{array}$ & \begin{tabular}{|l|} 
Tertile 2 \\
$16-27$ IU/L $(n=1791)$
\end{tabular} & $\begin{array}{l}\text { Tertile } 3 \\
\geq 28 \text { IU } / L(n=1711)\end{array}$ & \\
\hline Age, years & $53(49-58)$ & $53(49-59)$ & $54(50-59)$ & $53(49-57)$ & $<0.001$ \\
\hline Male sex, no. (\%) & $3486(68.1)$ & $618(38.2)$ & $1364(76.2)$ & $1504(87.9)$ & $<0.001$ \\
\hline Body mass index, $\mathrm{kg} / \mathrm{m}^{2}$ & $24.3(22.5-26.2)$ & $23.0(21.2-24.7)$ & $24.5(22.8-26.2)$ & $25.5(23.8-27.2)$ & $<0.001$ \\
\hline Waist circumference, $\mathrm{cm}$ & $85(80-90)$ & $80(75-86)$ & $86(80-90)$ & $89(84-93)$ & $<0.001$ \\
\hline Systolic blood pressure, $\mathrm{mmHg}$ & $119(110-128)$ & $115(107-124)$ & $119(110-128)$ & $122(113-130)$ & $<0.001$ \\
\hline Diastolic blood pressure, $\mathrm{mmHg}$ & $76(69-83)$ & $72(65-79)$ & $76(69-83)$ & $79(73-86)$ & $<0.001$ \\
\hline Diabetes mellitus, no. (\%) & $772(15.1)$ & $147(9.1)$ & $280(15.6)$ & $345(20.2)$ & $<0.001$ \\
\hline Hypertension, no. (\%) & $1778(34.7)$ & $406(25.1)$ & $634(35.4)$ & $738(43.1)$ & $<0.001$ \\
\hline Hyperlipidemia, no. (\%) & $1559(30.4)$ & $358(22.1)$ & $555(31.0)$ & $646(37.8)$ & $<0.001$ \\
\hline Current smoker, no. (\%) & $1024(20.0)$ & $136(8.4)$ & $371(20.7)$ & $517(30.2)$ & $<0.001$ \\
\hline Obesity, no. (\%) & $2114(41.3)$ & $362(22.4)$ & $756(42.3)$ & $996(58.2)$ & $<0.001$ \\
\hline $\begin{array}{l}\text { Family history of coronary artery } \\
\text { disease }^{\mathrm{a}} \text {, no. (\%) }\end{array}$ & $788(15.4)$ & $252(15.6)$ & $282(15.7)$ & $254(14.8)$ & 0.739 \\
\hline Fasting blood glucose, $\mathrm{mg} / \mathrm{dL}$ & $99(93-108)$ & $96(91-102)$ & $100(94-109)$ & $103(96-114)$ & $<0.001$ \\
\hline Total cholesterol, mg/dL & $193(172-217)$ & $190(171-214)$ & $192(171-214)$ & $198(176-221)$ & $<0.001$ \\
\hline $\begin{array}{l}\text { Low-density lipoprotein choles- } \\
\text { terol, } \mathrm{mg} / \mathrm{dL}\end{array}$ & $120(101-120)$ & $117(99-136)$ & $121(102-140)$ & $123(104-143)$ & $<0.001$ \\
\hline $\begin{array}{l}\text { High-density lipoprotein choles- } \\
\text { terol, } \mathrm{mg} / \mathrm{dL}\end{array}$ & $51(43-61)$ & $57(48-68)$ & $49(43-60)$ & $48(41-56)$ & $<0.001$ \\
\hline Triglycerides, mg/dL & $108(78-156)$ & $84(64-113)$ & $109(81-151)$ & $138(100-201)$ & $<0.001$ \\
\hline Creatinine, $\mathrm{mg} / \mathrm{dL}$ & $0.9(0.8-1.0)$ & $0.8(0.7-0.9)$ & $0.9(0.8-1.0)$ & $0.9(0.9-1.0)$ & $<0.001$ \\
\hline Uric acid, mg/dL & $5.5(4.5-6.4)$ & $4.7(3.9-5.6)$ & $5.6(4.8-6.4)$ & $6.0(5.2-7.0)$ & $<0.001$ \\
\hline Aspartate aminotransferase, IU/L & $25(21-31)$ & $22(19-26)$ & $24(21-29)$ & $28(24-36)$ & $<0.001$ \\
\hline Alanine aminotransferase, IU/L & $22(16-31)$ & $17(13-21)$ & $22(17-29)$ & $30(22-43)$ & $<0.001$ \\
\hline $\begin{array}{l}\text { High-sensitivity C-reactive pro- } \\
\text { tein } \geq 2 \mathrm{mg} / \mathrm{L}\end{array}$ & $47(0.9)$ & $7(0.4)$ & $21(1.2)$ & $19(1.1)$ & 0.046 \\
\hline
\end{tabular}

Table 1. Baseline characteristics of the study population according to the tertiles of gamma-glutamyl transferase. Values are presented as median (interquartile range) or number (\%). Pearson's chi-square test or Fisher's exact test were used for categorical variables and one-way analysis of variance or Kruskal-Wallis test were used for numerical variables. ${ }^{a}$ Coronary artery disease in a first-degree relative of any age.

\section{Results}

Baseline characteristics. All subjects were classified into tertiles based on GGT levels (Tertile $1 \leq 15$ IU/L, $\mathrm{n}=1618$; Tertile 2, 16-27 IU/L, $\mathrm{n}=1791$; Tertile 3, $\geq 28 \mathrm{IU} / \mathrm{L}, \mathrm{n}=1711$ ). The baseline characteristics of 5120 study participants according to the tertiles of GGT levels are summarized in Table 1 . The mean age of study participants was $53.8 \pm 7.6$ years, and 3486 (68.1\%) were males. The prevalence of males, diabetes mellitus, hypertension, hyperlipidemia, current smoking, and obesity was significantly higher in tertiles of increasing GGT levels. In addition, BMI, waist circumference, systolic and diastolic blood pressure, as well as levels of fasting blood glucose, total cholesterol, LDL cholesterol, triglyceride, uric acid, AST, and ALT increased with GGT category. In contrast, levels of HDL cholesterol were lower in tertiles of decreasing GGT levels.

CCTA findings. CCTA findings according to GGT tertiles are showed in Table 2. Mean CACS was $38.2 \pm 136.4$. Among the study participants, 405 (7.9\%) had significant stenosis of coronary artery $(\geq 50 \%$ stenosis diameter) in at least one coronary artery on CCTA. Mean CACS increased with GGT category $(p<0.001)$. A significant difference was presented in the prevalence of calcified, non-calcified, mixed, or any atherosclerotic plaques according to GGT level (all $p<0.001$ ). Scores which reflect plaque burden on CCTA such as segment involvement score, segment stenosis score, and modified Duke prognostic score increased with GGT tertile (all $p<0.001$ ). Furthermore, the prevalence of significant coronary artery stenosis, multi-vessel disease, and significant stenosis in the left main (LM) or proximal left anterior descending (LAD) artery also increased according to GGT tertile (all $p<0.001$ ).

Association between GGT levels and subclinical atherosclerosis. The association between GGT levels and subclinical atherosclerosis is described in Table 3. Univariable analyses revealed that increasing tertiles of GGT were significantly associated with subclinical coronary atherosclerosis detected by CCTA. After adjustment for cardiovascular risk factors (age, sex, obesity, diabetes mellitus, hypertension, hyperlipidemia, current smoking, family history of $\mathrm{CAD}$, and hs-CRP), no statistically significant differences were observed in the adjusted odds ratios (OR) for non-calcified and mixed plaques between GGT tertiles. The risk of atherosclerotic (OR 1.25, 95\% confidence interval [CI] 1.04-1.50, $p=0.016$ ) and calcified plaques (OR 1.24, 95\% CI: 1.02- 1.52, $p=0.033$ ), significant stenosis (OR 1.51,95\% CI: $1.10-2.07, p=0.011$ ), multi-vessel disease (OR 2.47, 95\% CI: 


\begin{tabular}{|c|c|c|c|c|c|}
\hline \multirow[b]{2}{*}{ Variables } & \multirow[b]{2}{*}{ Overall } & \multicolumn{3}{|c|}{ Gamma-glutamyl transferase } & \multirow[b]{2}{*}{$p$ value } \\
\hline & & Tertile 1 & Tertile 2 & Tertile 3 & \\
\hline Mean coronary artery calcium score & $38.2 \pm 136.4$ & $20.8 \pm 74.9$ & $42.6 \pm 152.2$ & $50.0 \pm 160.3$ & $<0.001$ \\
\hline Any atherosclerotic plaque, no. (\%) & $2083(40.7)$ & $471(29.1)$ & $778(43.4)$ & $834(48.7)$ & $<0.001$ \\
\hline \multicolumn{6}{|l|}{ Plaque characteristics, no. (\%) } \\
\hline Calcified plaque & $1371(26.8)$ & $300(18.5)$ & $521(29.1)$ & $550(32.1)$ & $<0.001$ \\
\hline Non-calcified plaque & $940(18.4)$ & $214(13.2)$ & $346(19.3)$ & $380(22.2)$ & $<0.001$ \\
\hline Mixed plaque & $451(8.8)$ & $91(5.6)$ & $164(9.2)$ & $196(11.5)$ & $<0.001$ \\
\hline Segment involvement score & $1.0 \pm 1.7$ & $0.7 \pm 1.4$ & $1.1 \pm 1.8$ & $1.3 \pm 1.9$ & $<0.001$ \\
\hline Segment stenosis score & $0.6 \pm 1.9$ & $0.3 \pm 1.2$ & $0.7 \pm 2.0$ & $0.8 \pm 2.3$ & $<0.001$ \\
\hline Modified Duke prognostic score & $1.2 \pm 0.6$ & $1.1 \pm 0.4$ & $1.2 \pm 0.7$ & $1.2 \pm 0.7$ & $<0.001$ \\
\hline \multicolumn{6}{|l|}{ Number of stenosed coronary arteries, no. (\%) } \\
\hline Significant stenosis & $405(7.9)$ & $78(4.8)$ & $146(8.2)$ & $181(10.6)$ & $<0.001$ \\
\hline One-vessel disease & $298(5.8)$ & $67(4.1)$ & $102(5.7)$ & $129(7.5)$ & $<0.001$ \\
\hline Multi-vessel disease & $107(2.1)$ & $11(0.7)$ & $44(2.5)$ & $52(3.0)$ & $<0.001$ \\
\hline Left main or proximal left anterior descending artery & $136(2.7)$ & $21(1.3)$ & $54(3.0)$ & $61(3.6)$ & $<0.001$ \\
\hline
\end{tabular}

Table 2. Comparison of coronary computed tomography angiographic findings according to the tertiles of gamma-glutamyltransferase. Values are presented as mean \pm standard deviation or number (\%). Pearson's chi-square test or Fisher's exact test were used for categorical variables and one-way analysis of variance or Kruskal-Wallis test were used for numerical variables.

\begin{tabular}{|c|c|c|c|c|}
\hline \multirow[b]{2}{*}{ Variables } & \multicolumn{2}{|l|}{ Univariable } & \multicolumn{2}{|l|}{ Multivariable } \\
\hline & Odds ratio $(95 \% \mathrm{CI})$ & $p$ value & Odds ratio $(95 \% \mathrm{CI})$ & $p$ value \\
\hline \multicolumn{5}{|c|}{ Any atherosclerotic plaque } \\
\hline Tertile 1 (reference) & 1 & - & 1 & - \\
\hline Tertile 2 & $1.87(1.62-2.16)$ & $<0.001$ & $1.09(0.92-1.29)$ & 0.348 \\
\hline Tertile 3 & $2.32(2.01-2.67)$ & $<0.001$ & $1.25(1.04-1.50)$ & 0.016 \\
\hline \multicolumn{5}{|l|}{ Calcified plaque } \\
\hline Tertile 1 (reference) & 1 & - & 1 & - \\
\hline Tertile 2 & $1.80(1.53-2.12)$ & $<0.001$ & $1.12(0.93-1.35)$ & 0.255 \\
\hline Tertile 3 & $2.08(1.77-2.45)$ & $<0.001$ & $1.24(1.02-1.52)$ & 0.033 \\
\hline \multicolumn{5}{|l|}{ Non-calcified plaque } \\
\hline Tertile 1 (reference) & 1 & - & 1 & - \\
\hline Tertile 2 & $1.57(1.31-1.89)$ & $<0.001$ & $1.06(0.87-1.30)$ & 0.560 \\
\hline Tertile 3 & $1.87(1.56-2.25)$ & $<0.001$ & $1.20(0.97-1.48)$ & 0.099 \\
\hline \multicolumn{5}{|l|}{ Mixed plaque } \\
\hline Tertile 1 (reference) & 1 & - & 1 & - \\
\hline Tertile 2 & $1.69(1.30-2.21)$ & $<0.001$ & $1.00(0.75-1.33)$ & 0.997 \\
\hline Tertile 3 & $2.17(1.68-2.81)$ & $<0.001$ & $1.17(0.87-1.58)$ & 0.306 \\
\hline \multicolumn{5}{|l|}{ Significant stenosis } \\
\hline Tertile 1 (reference) & 1 & - & 1 & - \\
\hline Tertile 2 & $1.75(1.32-2.33)$ & $<0.001$ & $1.15(0.85-1.56)$ & 0.370 \\
\hline Tertile 3 & $2.34(1.78-3.07)$ & $<0.001$ & $1.51(1.10-2.07)$ & 0.011 \\
\hline \multicolumn{5}{|l|}{ Multi-vessel disease } \\
\hline Tertile 1 (reference) & 1 & - & 1 & - \\
\hline Tertile 2 & $3.68(1.89-7.15)$ & $<0.001$ & $1.99(1.00-3.95)$ & 0.051 \\
\hline Tertile 3 & $4.58(2.38-8.81)$ & $<0.001$ & $2.47(1.22-4.98)$ & 0.012 \\
\hline \multicolumn{5}{|c|}{ Significant stenosis in the left main or proximal left anterior descending artery } \\
\hline Tertile 1 (reference) & 1 & -1 & 1 & - \\
\hline Tertile 2 & $2.36(1.42-3.93)$ & 0.001 & $1.63(0.95-2.78)$ & 0.074 \\
\hline Tertile 3 & $2.81(1.70-4.64)$ & $<0.001$ & $1.94(1.11-3.38)$ & 0.020 \\
\hline
\end{tabular}

Table 3. Association between gamma-glutamyl transferase levels and coronary computed tomography angiographic findings. CI confidence interval. Covariates in the multivariable model include age, sex, obesity, diabetes mellitus, hypertension, hyperlipidemia, creatinine, uric acid, current smoking, family history of coronary artery disease, and high-sensitivity C-reactive protein $\geq 2 \mathrm{mg} / \mathrm{L}$. 


\begin{tabular}{|c|c|c|c|c|}
\hline & $\begin{array}{l}\text { Tertile } 1 \\
(\mathrm{n}=1618)\end{array}$ & $\begin{array}{l}\text { Tertile } 2 \\
(\mathrm{n}=1791)\end{array}$ & $\begin{array}{l}\text { Tertile } 3 \\
(\mathrm{n}=1711)\end{array}$ & $p$ value $^{*}$ \\
\hline \multicolumn{5}{|l|}{ Cardiac event, no. (\%) } \\
\hline $\begin{array}{l}\text { Death/myocardial infarction/unstable angina requiring hospitalization/coronary } \\
\text { revascularization }\end{array}$ & $30(1.9)$ & $57(3.2)$ & $67(3.9)$ & 0.002 \\
\hline \multicolumn{5}{|l|}{ Clinical event, no. (\%) } \\
\hline Death & $20(1.2)$ & $17(0.9)$ & $15(0.9)$ & 0.510 \\
\hline Myocardial infarction & $1(0.1)$ & $2(0.1)$ & $0(0)$ & 0.396 \\
\hline Unstable angina requiring hospitalization & $0(0)$ & $1(0.1)$ & $9(0.5)$ & 0.001 \\
\hline Coronary revascularization & $10(0.6)$ & $38(2.1)$ & $52(3.0)$ & $<0.001$ \\
\hline Percutaneous coronary intervention & $10(0.6)$ & $37(2.1)$ & $49(2.9)$ & \\
\hline Coronary artery bypass surgery & $0(0)$ & $1(0.1)$ & $3(0.2)$ & \\
\hline Death/myocardial infarction/unstable angina requiring hospitalization & $21(1.3)$ & $19(1.1)$ & $24(1.4)$ & 0.624 \\
\hline
\end{tabular}

Table 4. Clinical outcomes according to the tertiles of gamma-glutamyl transferase. Values are presented as $\mathrm{n}$ (\%). $p$ values were calculated using the log-rank test* ${ }^{\star}$

\begin{tabular}{|c|c|c|c|c|c|c|c|c|}
\hline \multirow[b]{2}{*}{ Clinical outcomes } & \multicolumn{2}{|l|}{ Unadjusted } & \multicolumn{2}{|l|}{ Model 1} & \multicolumn{2}{|l|}{ Model 2} & \multicolumn{2}{|l|}{ Model 3} \\
\hline & HR (95\% CI) & $p$ value & HR (95\% CI) & $p$ value & HR (95\% CI) & $p$ value & HR (95\% CI) & $p$ value \\
\hline \multicolumn{9}{|l|}{ Cardiac events } \\
\hline Tertile 1 (reference) & 1 & - & 1 & - & 1 & - & 1 & - \\
\hline Tertile 2 & $1.71(1.10-2.66)$ & 0.017 & $1.39(0.88-2.20)$ & 0.155 & $1.34(0.85-2.12)$ & 0.207 & $1.35(0.86-2.12)$ & 0.192 \\
\hline Tertile 3 & $2.13(1.38-3.27)$ & 0.001 & $1.99(1.26-3.14)$ & 0.003 & $1.84(1.16-2.91)$ & 0.010 & $1.85(1.17-2.92)$ & 0.008 \\
\hline
\end{tabular}

Table 5. Univariable and multivariable analyses of gamma-glutamyl transferase levels for cardiac events, corrected for clinical risk factors. $H R$ hazards ratio; CI confidence interval. Model 1 was adjusted for age and sex; model 2 was adjusted further for diabetes mellitus, hypertension, hyperlipidemia, and current smoking; and model 3 was adjusted further for body mass index $\left(\mathrm{kg} / \mathrm{m}^{2}\right)$, creatinine, uric acid, family history of coronary artery disease, and high-sensitivity C-reactive protein $\geq 2 \mathrm{mg} / \mathrm{L}$.

$1.22-4.98, p=0.012$ ), and significant stenosis in the LM or proximal LAD artery (OR 1.94, 95\% CI: $1.11-3.38$, $p=0.020$ ) was higher in the third GGT tertile than in the first GGT tertile.

Clinical outcomes. During the follow-up period (median 5.4 years [interquartile range, 4.4-6.3 years]), a total of 165 cardiac events occurred in 154 patients: 52 all-cause deaths, three myocardial infarctions, 10 unstable angina requiring hospitalization, and 100 coronary revascularizations (Table 4). After adjustment for stepwise cardiovascular risk factors, the third GGT tertile had a significant adjusted hazard ratios for cardiac event, which was defined as a composite of all-cause death, myocardial infarction, unstable angina requiring hospitalization, or coronary revascularization, relative to that of the first GGT tertile (all $p<0.05$, Table 5).

\section{Discussion}

The main findings of the present study were as follows: (1) in asymptomatic individuals, elevated serum GGT levels were significantly associated with atherosclerotic plaques, especially calcified plaques, even after adjustment for cardiovascular risk factors; (2) high serum GGT levels were an independent predictor of significant coronary atherosclerosis such as significant stenosis in at least one coronary artery, multi-vessel disease, and significant stenosis in the LM or proximal LAD; (3) during a follow-up of median 5.4 years, individuals with high serum GGT levels experienced more cardiac events.

The association between elevated GGT levels and risk of CAD or CVD has been suggested in large epidemiologic studies ${ }^{4-6}$. Subsequent studies have reported that higher GGT levels were significantly associated with endothelial dysfunction, carotid artery plaques, and arterial stiffness ${ }^{13-15}$. Moreover, recent studies have indicated that GGT levels were significantly related to high CACS and its progression, which reflects total atherosclerotic burden and is a surrogate marker of future CAD events ${ }^{11,12}$. However, the absence of coronary calcification does not exclude the presence of clinically significant and potentially vulnerable atherosclerotic plaques ${ }^{16,17}$. Although CCTA provides more comprehensive information regarding coronary atherosclerosis, there are limited data on the association between GGT and subclinical coronary atherosclerosis evaluated by CCTA. An observational cohort study with CCTA investigated the association between GGT levels and coronary atherosclerotic plaques ${ }^{18}$. However, it analyzed limited data without clinical outcomes. Therefore, the present study aimed to evaluate the influence of GGT levels on the risk of subclinical coronary atherosclerosis on CCTA and cardiac outcomes.

In the present study, individuals with higher GGT levels had a higher prevalence, extent, and severity of coronary atherosclerosis detected by CCTA. Even after adjustments for clinical and laboratory variables, higher GGT levels were an independent predictor of coronary atherosclerotic plaques, especially calcified plaques. 
Thus, our results were consistent with previous studies evaluating CACS ${ }^{11,12}$. Notably, higher GGT levels were an independent risk factor for high-risk feature CAD such as multi-vessel disease and significant stenosis in the LM or proximal LAD, which are known to be associated with poorer prognosis ${ }^{19}$. As a result, in the current study, individuals with higher GGT levels experienced more cardiac events. Therefore, our findings suggest that subjects with high serum GGT levels may benefit from preemptive cardiovascular risk evaluation and potentially, regular cardiovascular risk surveillance to guard against future cardiovascular events.

Although the exact mechanisms underpinning the association between serum GGT and CVD remain unclear, one potential pathophysiology is that they may be linked via NAFLD. Clinically, GGT is a hepatic enzyme that reflects the degree of hepatic inflammation or fibrosis ${ }^{20}$ and has been suggested as a surrogate marker for NAFLD ${ }^{2,3}$. NAFLD is regarded as the hepatic manifestation of metabolic syndrome and insulin resistance, which may increase the risk of atherosclerosis ${ }^{7,8,21}$. A chronic activation of systemic and hepatic inflammation may also act as a common pathway for NAFLD and CVD ${ }^{22}$. NAFLD is progressive across simple fatty livers to steatohepatitis, fibrosis, and finally liver cirrhosis. In particular, steatohepatitis with the potential of hepatic inflammation or fibrosis has been reported to increase the risk of cardiovascular mortality and morbidity ${ }^{23-25}$. To diagnose NAFLD, a liver biopsy is required which has several limitations including invasiveness, complications, sampling variability, and $\cos ^{26,27}$. Ultrasonography typically usually employed as a substitute for liver biopsy, but there is a limit to the distinction between simple fatty liver and steatohepatitis ${ }^{28,29}$. Instead, noninvasive methods to determine the presence of hepatic inflammation or fibrosis have been developed. Of these, NAFLD fibrosis score (NFS) and Fibrosis-4 (FIB-4) are representative scoring systems that have been well-validated ${ }^{30,31}$. NFS include factors representing metabolic syndrome such as BMI or impaired glucose tolerance/diabetes mellitus, while FIB-4 includes age and serum markers. Although several studies have presented a significant association between NFS or FIB-4 and the prevalence or future risk of CVD, the results were not fully adjusted for other cardiovascular risk factors ${ }^{32-34}$. This study sought to evaluate the relationship between GGT levels as a noninvasive marker for hepatic inflammation or fibrosis and characteristics of atherosclerosis with their prognosis in a large cohort of asymptomatic Korean individuals who voluntarily underwent CCTA, which has proven to be effective in providing a comprehensive evaluation of coronary atherosclerosis ${ }^{10}$.

Our study has several limitations. First, the present study was performed at a single center, and all study participants voluntarily visited the hospital for a general health examination. Therefore, there was a potential of selection bias. Second, our study participants were exclusively Korean, limiting the applicability of our findings to other ethnic groups. Third, as the current study was a retrospective cohort study, these data may not fully reflect patient outcomes. Additionally, we did not specify the cause of death. Fourth, calcified plaques and higher CACS may lead to overestimation of significant coronary artery stenosis. Finally, CCTA has potential drawbacks, including radiation hazards, use of contrast, and higher cost. Therefore, although this study enrolled only volunteers, the use of CCTA in asymptomatic individuals cannot be justified.

In conclusion, this large retrospective cohort study in asymptomatic individuals undergoing CCTA demonstrated that elevated serum GGT levels were independently associated with high-risk subclinical atherosclerosis, resulting in poorer cardiac outcomes. Therefore, cardiovascular risk evaluation and surveillance should be considered in subjects with high GGT levels to prevent future cardiovascular disease. These findings should be further investigated and validated in future studies.

\section{Materials and methods}

Study population. This was a retrospective observational cohort study which analyzed 9269 consecutive South Korean individuals aged $\geq 20$ years who had undergone self-referred CCTA evaluation as part of a general health examination at the Health Screening and Promotion Center in the Asan Medical Center between January 2007 and December 2011. Among these, 7129 (76.9\%) agreed to participate in the present study. Possible risks associated with CCTA were explained, and written informed consent was obtained from each participant. Exclusion criteria included subjects with (1) a previous history of significant alcohol intake with $\geq 210 \mathrm{~g} /$ week in males and $140 \mathrm{~g} /$ week in females ${ }^{35,36}$; (2) a previous history of angina or myocardial infarction; (3) abnormal resting electrocardiography results, i.e., pathological Q waves, ischemic ST segments or T-wave changes, or left bundlebranch blocks; (4) insufficient medical records; (5) structural heart disease; (6) unmeasured GGT; (7) a previous cardiac procedure; (8) a previous history of open-heart surgery or percutaneous coronary intervention; or (9) renal insufficiency (creatinine $>1.5 \mathrm{mg} / \mathrm{dL}$ ). A final total of 5120 subjects were enrolled (Fig. 1). The study was approved by the local Institutional Review Board of the Asan Medical Center, Seoul, Korea (2016-1068). Written informed consent under the 'Ethics, consent and permissions' was obtained from each participant. This study was carried out in accordance with Good Clinical Practice (GCP) guidelines and the Declaration of Helsinki.

Clinical and laboratory measurements. Basic demographic data of study participants were acquired from a database maintained by the Health Screening and Promotion Center at the Asan Medical Center. Medical history including angina, myocardial infarction, stroke, structural heart disease, open heart surgery, percutaneous coronary intervention, previous cardiac procedures, diabetes mellitus, hypertension, hyperlipidemia, or smoking status, was obtained from the responses in the systemized self-report questionnaire issued prior to the general health examination ${ }^{37}$.

Height and weight were obtained with subjects wearing light clothing and no shoes. Body mass index (BMI) was calculated as weight in kilograms divided by the square of height in meters $\left(\mathrm{kg} / \mathrm{m}^{2}\right)$. Waist circumference $(\mathrm{cm})$ was measured mid-way between the lower costal margin and iliac crest at the end of a normal expiration of breath by a well-trained nurse. Blood pressure was measured on the right arm after $a \geq 5$ min rest using an automatic manometer and an appropriate cuff size. After overnight fasting, early morning blood samples were drawn from the antecubital vein into vacuum tubes and subsequently analyzed in the central certified laboratory 


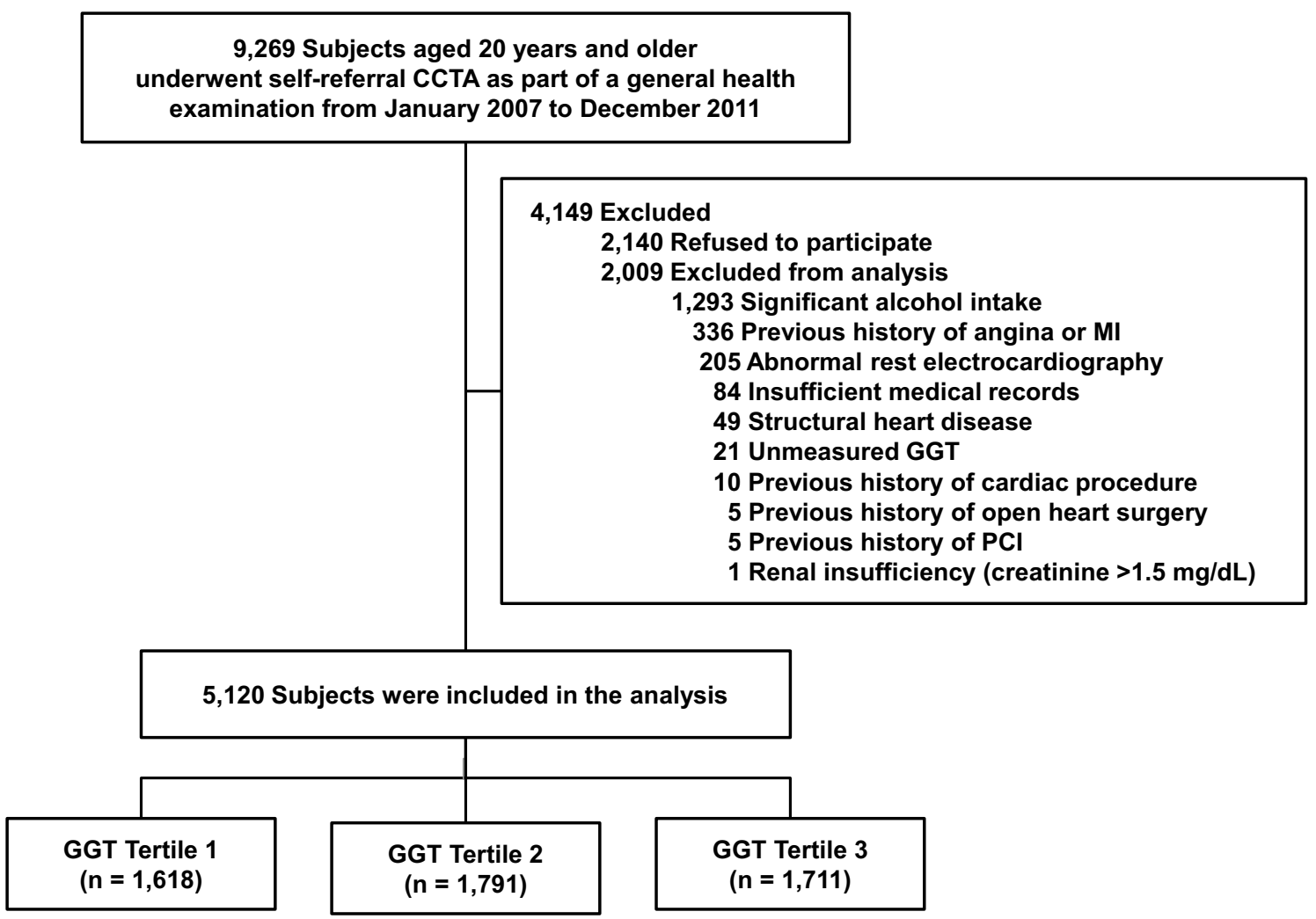

Figure 1. Overview of the study population. CCTA coronary computed tomographic angiography; GGT gamma-glutamyl transferase; $M I$ myocardial infarction; PCI percutaneous coronary intervention.

of the Asan Medical Center. The concentrations of GGT, aspartate aminotransferase (AST), alanine aminotransferase (ALT), fasting plasma glucose, creatinine, uric acid, fasting total cholesterol, triglycerides, high-density lipoprotein (HDL) cholesterol, low-density lipoprotein (LDL) cholesterol, and high-sensitivity C-reactive protein (hs-CRP) were measured ${ }^{38}$.

Obesity was defined as a BMI $\geq 25 \mathrm{~kg} / \mathrm{m}^{2}$ on the basis of an Asian-specific cutoff point as recommended in the World Health Organization. Diabetes mellitus was defined as subjects with a self-reported history of diabetes and/or treatment with dietary modification, use of anti-diabetic medication as indicated on the systemized questionnaire, or those with a fasting plasma glucose $\geq 126 \mathrm{mg} / \mathrm{dL}$. Hypertension was defined as blood pressure $\geq 140 / 90 \mathrm{mmHg}$ or a self-reported history of hypertension and/or use of anti-hypertensive medication. Hyperlipidemia was defined as total cholesterol $\geq 240 \mathrm{mg} / \mathrm{dL}$ or use of an anti-hyperlipidemic medication. A family history of CAD was defined as having a first-degree relative of any age with CAD based on the self-report questionnaire $^{37}$.

CCTA image acquisition and analysis. CCTA was conducted using either single-source 64-slice CT (LightSpeed VCT, GE, Milwaukee, WI, USA) or dual-source CT (Somatom Definition, Siemens, Erlangen, Germany). A standard scanning protocol was used, as previously described ${ }^{10}$. All CCTA scans were analyzed using a dedicated workstation (Advantage Workstation, GE; or Volume Wizard, Siemens) by experienced cardiovascular radiologists (DHY, JWK, and THL). According to the guidelines of the Society of Cardiovascular Computed Tomography, a 16-segment coronary artery tree model was used ${ }^{39}$. A coronary artery calcium score (CACS) was measured and categorized by scores of 0,1 to 10,11 to 100,101 to 400 , and $>400^{40}$. Plaques containing calcified tissue involving more than $50 \%$ of the plaque area (density $>130 \mathrm{HU}$ ) were classified as calcified, plaques with $<50 \%$ calcium were classified as mixed, and plaques without calcium were classified as non-calcified lesions ${ }^{41}$. The contrast-enhanced portion of the coronary lumen was semi-automatically traced at the site of maximal stenosis and compared with the mean value of the proximal and distal reference sites. Stenosis $\geq 50 \%$ was defined as significant ${ }^{10}$. The overall plaque burden was determined from coronary artery plaque scores calculated from modified Duke prognostic scores, segment stenosis scores, and segment involvement scores, as described previously ${ }^{42}$.

Clinical outcomes. Follow-up clinical data were obtained by a review of medical records or telephone interviews using trained personnel through to the end of June 2017. A cardiac event was defined as a composite of all-cause death, myocardial infarction, unstable angina requiring hospitalization, or coronary revascularization. The diagnosis of myocardial infarction was based on the presence of new Q waves in at least two contiguous leads, or an elevation of creatine kinase or its myocardial band isoenzyme to at least three times the upper limit 
of the normal range at follow-up. Revascularization was performed if there was a stenosis of at least $50 \%$ of the diameter noted on invasive coronary angiography with a positive stress test result or if there was a stenosis of at least $70 \%$ observed on invasive coronary angiography ${ }^{43}$.

Statistical analysis. Categorical variables are expressed as frequencies with percentages. Continuous variables are expressed as the mean and standard deviation. Between-group comparisons were performed using Pearson's chi-square test or Fisher's exact test for categorical variables and one-way analysis of variance or Kruskal-Wallis test for numerical variables, as appropriate. Univariable and multivariable analyses were performed using a logistic regression model to analyze the association between serum GGT levels and subclinical coronary atherosclerosis on CCTA. Based on previous epidemiologic studies ${ }^{44,45}$, we selected covariates in the multivariable model according to their clinical importance and statistical significance, which included age, sex, obesity, diabetes mellitus, hypertension, hyperlipidemia, creatinine, uric acid, current smoking, family history of $\mathrm{CAD}$, and hs-CRP $\geq 2 \mathrm{mg} / \mathrm{L}$. Unadjusted and adjusted odds ratios with $95 \%$ confidence intervals for the logistic regression were calculated. To investigate the associations of serum GGT levels and cardiac events, we also conducted the Cox proportional hazards regression analyses with adjustment for potential confounders. Stepwise multivariable models were determined by the backward variable selection approach. All reported $p$ values are two-sided, and $p<0.05$ was considered statistically significant. Data manipulation and statistical analyses were performed using SPSS software (Version 18; SPSS Inc., Chicago, IL, USA).

\section{Data availability}

The datasets used and/or analyzed during the current study available from the corresponding author on request.

Received: 21 May 2020; Accepted: 12 October 2020

Published online: 22 October 2020

\section{References}

1. Whitfield, J. B. Gamma glutamyl transferase. Crit. Rev. Clin. Lab. Sci. 38, 263-355 (2001).

2. Angulo, P. Nonalcoholic fatty liver disease. N. Engl. J. Med. 346, 1221-1231 (2002).

3. Hossain, I. A., Rahman, S. M. M., Rahman, M. K. \& Ali, L. Gamma glutamyl transferase is an independent determinant for the association of insulin resistance with nonalcoholic fatty liver disease in Bangladeshi adults: Association of GGT and HOMA-IR with NAFLD. Diabetes Metab. Syndr. 10, 25-29 (2016).

4. Lee, D. S. et al. Gamma glutamyl transferase and metabolic syndrome, cardiovascular disease, and mortality risk: the Framingham heart study. Arterioscler. Thromb. Vasc. Biol. 27, 127-133 (2007).

5. Strasak, A. M. et al. Longitudinal change in serum gamma-glutamyltransferase and cardiovascular disease mortality: a prospective population-based study in 76,113 Austrian adults. Arterioscler. Thromb. Vasc. Biol. 28, 1857-1865 (2008).

6. Wannamethee, G., Ebrahim, S. \& Shaper, A. G. Gamma-glutamyltransferase: determinants and association with mortality from ischemic heart disease and all causes. Am. J. Epidemiol. 142, 699-708 (1995).

7. Kozakova, M. et al. Fatty liver index, gamma-glutamyltransferase, and early carotid plaques. Hepatology 55, 1406-1415 (2012).

8. Ndrepepa, G., Colleran, R. \& Kastrati, A. Gamma-glutamyl transferase and the risk of atherosclerosis and coronary heart disease. Clin. Chim. Acta. 476, 130-138 (2018).

9. Targher, G. \& Byrne, C. D. Circulating markers of liver function and cardiovascular disease risk. Arterioscler. Thromb. Vasc. Biol. 35, 2290-2296 (2015).

10. Park, G. M. et al. Prevalence of coronary atherosclerosis in an Asian population: findings from coronary computed tomographic angiography. Int. J. Cardiovasc. Imaging. 31, 659-668 (2015).

11. Cho, Y. K. et al. Association between serum gamma-glutamyltransferase and the progression of coronary artery calcification. Atherosclerosis 243, 300-306 (2015).

12. Atar, A. I. et al. Association between gamma-glutamyltransferase and coronary artery calcification. Int. J. Cardiol. 167, 1264-1267 (2013).

13. Arinc, H. et al. Serum gamma glutamyl transferase and alanine transaminase concentrations predict endothelial dysfunction in patients with non-alcoholic steatohepatitis. Ups. J. Med. Sci. 118, 228-234 (2013).

14. Toshikuni, N. et al. Elevated serum gamma-glutamyl transpeptidase levels and fatty liver strongly predict the presence of carotid plaque. J. Atheroscler. Thromb. 22, 1051-1060 (2015).

15. Jung, C. H. et al. Serum gamma-glutamyltransferase is associated with arterial stiffness in healthy individuals. Clin. Endocrinol. (Oxf.) 75, 328-334 (2011).

16. van Velzen, J. E. et al. Comparison of the relation between the calcium score and plaque characteristics in patients with acute coronary syndrome versus patients with stable coronary artery disease, assessed by computed tomography angiography and virtual histology intravascular ultrasound. Am. J. Cardiol. 108, 658-664 (2011).

17. Yoon, Y. E. et al. The absence of coronary artery calcification does not rule out the presence of significant coronary artery disease in Asian patients with acute chest pain. Int. J. Cardiovasc. Imaging. 28, 389-398 (2012).

18. Cho, H. S. et al. Clinical significance of serum bilirubin and gamma-glutamyltransferase levels on coronary atherosclerosis assessed by multidetector computed tomography. Nutr. Metab. Cardiovasc. Dis. 25, 677-685 (2015).

19. Min, J. K. et al. All-cause mortality benefit of coronary revascularization vs. medical therapy in patients without known coronary artery disease undergoing coronary computed tomographic angiography: results from CONFIRM (COronary CT angiography evaluation for clinical outcomes: an international multicenter registry). Eur. Heart J. 33, 3088-3097 (2012).

20. Korkmaz, H., Unler, G. K., Gokturk, H. S., Schmidt, W. E. \& Kebapcilar, L. Noninvasive estimation of disease activity and liver fibrosis in nonalcoholic fatty liver disease using anthropometric and biochemical characteristics, including insulin, insulin resistance, and 13C-methionine breath test. Eur. J. Gastroenterol. Hepatol. 27, 1137-1143 (2015).

21. Loria, P., Lonardo, A. \& Targher, G. Is liver fat detrimental to vessels?: intersections in the pathogenesis of NAFLD and atherosclerosis. Clin. Sci. (Lond.) 115, 1-12 (2008).

22. Schindhelm, R. K. et al. Alanine aminotransferase predicts coronary heart disease events: a 10-year follow-up of the Hoorn Study. Atherosclerosis. 191, 391-396 (2007).

23. Ekstedt, M. et al. Long-term follow-up of patients with NAFLD and elevated liver enzymes. Hepatology 44, 865-873 (2006).

24. Kim, D., Kim, W. R., Kim, H. J. \& Therneau, T. M. Association between noninvasive fibrosis markers and mortality among adults with nonalcoholic fatty liver disease in the United States. Hepatology 57, 1357-1365 (2013).

25. Chen, Y. et al. Advanced fibrosis associates with atherosclerosis in subjects with nonalcoholic fatty liver disease. Atherosclerosis. 241, 145-150 (2015) 
26. Bedossa, P. \& Carrat, F. Liver biopsy: the best, not the gold standard. J. Hepatol. 50, 1-3 (2009).

27. Emanuele, E. Is biopsy always necessary? Toward a clinico-laboratory approach for diagnosing nonalcoholic steatohepatitis in obesity. Hepatology 48, 2086-2087 (2008).

28. Charatcharoenwitthaya, P. \& Lindor, K. D. Role of radiologic modalities in the management of non-alcoholic steatohepatitis. Clin. Liver Dis. 11, 37-54 (2007).

29. Li, Q., Dhyani, M., Grajo, J. R., Sirlin, C. \& Samir, A. E. Current status of imaging in nonalcoholic fatty liver disease. World J. Hepatol. 10, 530-542 (2018).

30. Angulo, P. et al. The NAFLD fibrosis score: a noninvasive system that identifies liver fibrosis in patients with NAFLD. Hepatology 45, 846-854 (2007).

31. Sterling, R. K. et al. Development of a simple noninvasive index to predict significant fibrosis in patients with $\mathrm{HIV} / \mathrm{HCV}$ coinfection. Hepatology 43, 1317-1325 (2006).

32. Onnerhag, K., Hartman, H., Nilsson, P. M. \& Lindgren, S. Non-invasive fibrosis scoring systems can predict future metabolic complications and overall mortality in non-alcoholic fatty liver disease (NAFLD). Scand. J. Gastroenterol. 54, 328-334 (2019).

33. Parikh, N. S., VanWagner, L. B., Elkind, M. S. V. \& Gutierrez, J. Association between nonalcoholic fatty liver disease with advanced fibrosis and stroke. J. Neurol. Sci. 407, 116524 (2019).

34. Song, D. S., Chang, U. I., Kang, S. G., Song, S. W. \& Yang, J. M. Noninvasive serum fibrosis markers are associated with coronary artery calcification in patients with nonalcoholic fatty liver disease. Gut Liver 13, 658-668 (2019).

35. Chalasani, N. et al. The diagnosis and management of non-alcoholic fatty liver disease: practice guideline by the American Association for the Study of Liver Diseases, American College of Gastroenterology, and the American Gastroenterological Association. Hepatology 55, 2005-2023 (2012).

36. O'Shea, R. S., Dasarathy, S. \& McCullough, A. J. Practice guideline Committee of the American Association for the Study of Liver D, Practice Parameters Committee of the American College of G alcoholic liver disease. Hepatology 51, 307-328 (2010).

37. Park, G. M. et al. Model for assessing cardiovascular risk in a Korean population. Circ. Cardiovasc. Qual. Outcomes. 7, 944-951 (2014).

38. Park, G. M. et al. Family history of diabetes and the risk of subclinical atherosclerosis. Diabetes Metab. 42, 170-177 (2016).

39. Raff, G. L. et al. SCCT guidelines for the interpretation and reporting of coronary computed tomographic angiography. J. Cardiovasc. Comput. Tomogr. 3, 122-136 (2009).

40. Agatston, A. S. et al. Quantification of coronary artery calcium using ultrafast computed tomography. J. Am. Coll. Cardiol. 15, 827-832 (1990).

41. Leber, A. W. et al. Accuracy of 64-slice computed tomography to classify and quantify plaque volumes in the proximal coronary system: a comparative study using intravascular ultrasound. J. Am. Coll. Cardiol. 47, 672-677 (2006).

42. Min, J. K. et al. Prognostic value of multidetector coronary computed tomographic angiography for prediction of all-cause mortality. J. Am. Coll. Cardiol. 50, 1161-1170 (2007).

43. Group BDS. A randomized trial of therapies for type 2 diabetes and coronary artery disease. N. Engl. J. Med. 360, 2503-2515 (2009).

44. Goff, D. C. Jr. et al. 2013 ACC/AHA guideline on the assessment of cardiovascular risk: a report of the American College of Cardiology/American Heart Association Task Force on practice guidelines. J. Am. Coll. Cardiol. 63, 2935-2959 (2014).

45. Conroy, R. M. et al. Estimation of ten-year risk of fatal cardiovascular disease in Europe: the SCORE project. Eur. Heart J. 24, 987-1003 (2003).

\section{Author contributions}

Y.G.K., S.B.L., and G.M.P. were responsible for the concept and design of the study, data acquisition, analysis and interpretation, and drafting of the manuscript. D.H.Y., J.W.K., and T.H.L. analyzed the coronary computed tomography angiography. H.K.K., J.C., S.W.L., and Y.H.K. critically revised the manuscript and provided important intellectual content.

\section{Funding}

This research was supported by the Basic Science Research Program through the National Research Foundation of Korea funded by the Ministry of Education (2018R1D1A3B07043344).

\section{Competing interests}

The authors declare no competing interests.

\section{Additional information}

Correspondence and requests for materials should be addressed to G.-M.P. or S.B.L.

Reprints and permissions information is available at www.nature.com/reprints.

Publisher's note Springer Nature remains neutral with regard to jurisdictional claims in published maps and institutional affiliations.

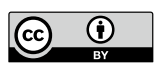

Open Access This article is licensed under a Creative Commons Attribution 4.0 International License, which permits use, sharing, adaptation, distribution and reproduction in any medium or format, as long as you give appropriate credit to the original author(s) and the source, provide a link to the Creative Commons licence, and indicate if changes were made. The images or other third party material in this article are included in the article's Creative Commons licence, unless indicated otherwise in a credit line to the material. If material is not included in the article's Creative Commons licence and your intended use is not permitted by statutory regulation or exceeds the permitted use, you will need to obtain permission directly from the copyright holder. To view a copy of this licence, visit http://creativecommons.org/licenses/by/4.0/.

(C) The Author(s) 2020 